\title{
UM LEVANTAMENTO BIBLIOGRÁFICO SOBRE ALFABETIZAÇÃo CIENTÍFICA A PARTIR DO GOOGLE ACADÊMICO
}

\author{
A Biblography Data SuRVEY ABOUT SCIENTIFIC LITERACy \\ THROUGH ACADEMIC GOOGLE
}

DOI: http://dx.doi.org/10.23926/RPD.2526-2149.2019.v4.n2.p641-652.id493

\section{Sara Barbosa dos \\ Santos \\ Graduanda em Biologia \\ (IFMA) \\ sara201498@hotmail.com}

\section{Elson Silva de Sousa}

Doutorando em Educação em

Ciências e Matemática

(UFPA/REAMEC)

Professor no Instituto Federal

do Maranhão (IFMA)

elson.silva@ifma.edu.br

\section{Rogério Soares \\ Cordeiro}

Doutor em Biotecnologia

(UMC)

Professor no Instituto Federal do Maranhão (IFMA)

rogerio.cordeiro@ifma.edu.br

\section{Jesuíno da Silva Costa Martins}

Mestre em Genética e

Melhoramento (UFPI)

Professor no Instituto Federal

do Maranhão (IFMA)

jesuino.martins@ifma.edu.br
Resumo: A Alfabetização Científica (AC) vem sendo apontada na literatura como condição fundamental para que os indivíduos desenvolvam competências e habilidades necessárias para agir de forma crítica, consciente e autônoma na sociedade contemporânea. Partindo disso, este trabalho, do tipo bibliométrico, objetivou traçar um panorama sobre a temática da AC por meio do levantamento bibliográfico da produção acadêmica disponibilizada gratuitamente no Google Acadêmico, destacando a evolução cronológica, as principais fontes de publicação, os autores que mais produziram e as palavras-chave mais citadas. A pesquisa possibilitou gerar evidências generalizáveis sobre a Alfabetização Científica, conhecer o perfil da produção acadêmica sobre a temática e fornecer subsídios para trabalhos futuros sobre o assunto. Identificou-se que houve um salto na publicação de trabalhos sobre a temática a partir dos anos 2000. É expressiva a multiplicidade de fontes onde os trabalhos vêm sendo publicados. Há um pequeno grupo de autores com tradição de publicação na área, considerando a significativa quantidade de autores associados a apenas um único artigo. O conjunto de palavras-chave extraídas apresentou alta variedade e os três autores com maior número de publicação, na amostra analisada, foram: Sasseron, Lorenzetti, Fachín-Terán.

Palavras-chave: Ensino de Ciências; Base de dados; Bibliometria; Google Scholar.

\begin{abstract}
The Scientific Literacy has been being nominated in literature as a fundamental tool to the individuals who develop competences and necessary abilities in order to act critical, conscious and autonomously in the present society. This bibliometric work has had as its objective to map an outlook about the Scientific Literacy through the bibliographic survey of the academic production available free of charge at Academic Google. This study highlights the chronological evolution, the main publishing sources, the authors who have produced and the cited key words. The research has enabled to generate generalizable evidence of the Scientific Literacy, to know the profile of the academic production about the topic and to provide grants to future studies. There has been an increasing number of publications about this topic from 2000 on. There is a great variety of sources published about this topic. There is a small group of authors with tradition of studies published in the area, considering the significant quantity of authors related to an only one article. The set of key words extracted has shown a great variety and three common writers were Sasseron, Lorenzetti, Fachín-Terán.

Keywords: Science Teaching, Data Collection, Bibliometry, Google Scholar.
\end{abstract}




\section{INTRODUÇÃO}

Nas duas últimas décadas, a Alfabetização Científica (AC) tem-se destacado como uma das mais importantes vertentes vinculadas ao processo de ensino-aprendizagem em Ciências. Diversos pesquisadores brasileiros têm estudado a $\mathrm{AC}$ em diferentes níveis e modalidades de ensino com enfoques e objetivos diversos (AULER; DELIZOICOV, 2001; BRANDI; GURGEL, 2002; CHASSOT, 2003; LORENZETTI; DELIZOICOV, 2001; PRAIA; GILPÉREZ; VILCHES, 2007; SASSERON; CARVALHO, 2011; TEIXEIRA， 2013; VIECHENESKI; LORENZETTI; CARLETTO, 2015).

A ciência escolar tem o objetivo de "ajudar os estudantes a alcançar níveis mais altos de Alfabetização Científica" (BYBEE, 1995, p. 28, tradução nossa). A Alfabetização Científica (AC) considera o desenvolvimento de competências e habilidades necessárias para as pessoas serem capazes de agir de forma crítica, consciente e autônoma na sociedade contemporânea e no mundo. Ela aponta para uma ciência que deve ser ensinada de modo a realizar com os alunos, a aplicação do conhecimento científico em suas vidas diárias. Está apoiada na ideia de um ensino-aprendizagem da ciência que contribua para atingir fins educativos reais, tais como o desenvolvimento da imaginação criativa, pensamento crítico e que promova a educação global dos alunos (AULER; DELIZOICOV, 2001).

Face ao exposto, e considerando a relevância da temática apresentada, este trabalho partiu das seguintes questões norteadoras: Qual é o perfil histórico bibliométrico da produção científica acadêmica sobre a Alfabetização Científica disponível no Google Acadêmico? Quais são as principais fontes de publicação? Quais autores com maior produção e as palavras-chave mais citadas nesses trabalhos?

O Google Acadêmico, desenvolvido pela Google Inc. e lançado no final de 2004, temse apresentado como uma ferramenta de pesquisa em produção científica bastante promissora. Meho e Yang (2007), Harzing e Wal (2008), Kousha e Thelwall (2011) realizaram estudos sobre o Google Acadêmico, inclusive em comparação com outras bases de dados - Web of Science e Scopus - e consideraram que o Google Acadêmico fornece uma visão abrangente potencialmente útil da produção científica disponível e, por vezes, supera bases de dados referenciais reconhecidas internacionalmente. O objetivo do Google Acadêmico é fornecer acesso à literatura mundial acadêmica, tanto de registros bibliográficos de seu principal mecanismo de busca, quanto de outras bases de dados e sistemas de informação.

Nesse contexto, o estudo objetivou mapear a produção científica acadêmica sobre a Alfabetização Científica, a partir da base de dados disponível gratuitamente no Google 
Acadêmico, buscando traçar o perfil dos artigos sobre a temática, destacando a evolução histórica e as principais fontes de publicação, os autores que mais produziram e as palavraschave mais citadas.

\section{Alfabetização Científica e a Literatura NaCiOnal}

A discussão sobre a Alfabetização Científica (AC) tem tomado cada vez mais espaço na nossa sociedade, apresentando conceitos e significados muito amplos e distintos para vários escritores da literatura brasileira. Para Soares, por exemplo, a AC "é o estado ou condição que assume aquele que aprende a ler e escrever" (SOARES, 1998, p. 17), enquanto para Fourez ela representa "um tipo de saber, de capacidade ou de conhecimento e de saber-ser que, em nosso mundo técnico-científico, seria uma contraparte ao que foi a alfabetização no último século" (FOUREZ, 1995).

A Alfabetização Científica é importante à medida que - juntamente a outros conhecimentos importantes - ajuda o ser humano a atuar e viver no mundo. Por isso, deve ser acompanhada de uma formação humana crítica (FOUREZ, 1995).

Alguns pesquisadores nacionais utilizam o termo "Letramento Científico" apoiando-se no significado do termo de duas pesquisadoras. Para Soares (1998, p. 18), o letramento é o "resultado da ação de ensinar ou aprender a ler e escrever: estado ou condição que adquire um grupo social ou um indivíduo como consequência de ter-se apropriado da escrita”. Já para Kleiman (1995) Letramento "é o conjunto de práticas sociais que usam a escrita enquanto sistema simbólico e enquanto tecnologia, em contextos específicos para objetivos específicos” (p. 19).

É relevante destacar que alguns autores brasileiros utilizam o termo "Enculturação Científica" partindo da ideia de que o ensino da ciência promova aos alunos a sensação de que as ideias e conceitos científicos são parte de seu corpus.

$\mathrm{Na}$ literatura científica acadêmica, há trabalhos que se debruçaram em realizar levantamentos bibliográficos a fim de apresentar o estado da arte, estado do conhecimento ou dados bibliométricos da pesquisa em Alfabetização Científica.

A monografia intitulada Alfabetização Científica, buscou caracterizar e conceituar a temática a partir de um levantamento bibliográfico em duas bases de dados, Lisa e Web of Science, que retornaram 12 resultados positivos. O trabalho considerou que a AC "é um processo em constante mudança e renovação e abrange o entendimento/familiarização de 
conceitos científicos, bem como a habilidade de transformar esses conceitos em práticas" (GARRIDO, 2010).

Os pesquisadores Viecheneski, Lorenzetti e Carletto (2015) analisaram 25 artigos, envolvendo AC nos anos iniciais do Ensino Fundamental, publicados em nove edições do Encontro Nacional de Pesquisa em Educação em Ciências - ENPEC, do período entre 1997 a 2013. O estudo elencou o problema, as concepções, a metodologia, os objetivos, as propostas de promoção e as contribuições da AC para a Educação em Ciências e indicou que a "AC nos anos iniciais é uma área ainda incipiente nos anais dos ENPECs, apesar disso, os trabalhos que tratam do tema trazem contribuições significativas, indicando o potencial que a matéria agrega ao ensino de ciências e à formação de professores" (VIECHENESKI; LORENZETTI; CARLETTO, 2015).

Os resultados do levantamento bibliográfico realizado a partir dos trabalhos publicados no Encontro Nacional de Pesquisa em Educação em Ciência (ENPEC, 1997-2009) e Encontro de Pesquisa em Ensino de Física (EPEF, 1998 a 2008) apontaram para "a heterogeneidade de perspectivas e concepções acerca do tema Alfabetização e letramento científico-tecnológico e que o número de trabalhos aumentou em cada um desses eventos" (MION; ANJOS; PIAZZETTA, 2010).

Outro estudo, ainda mais recente, buscou caracterizar o estado do conhecimento na área da literacia em diferentes contextos da educação nacional e internacional a partir das produções publicadas entre 2005 a 2015 na base de dados Web Scopus. Os nove trabalhos analisados convergiram para a "importância das práticas da literacia no contexto da educação e da sociedade [...] como elemento potencializador de oportunidades para que os sujeitos envolvidos no processo possam mobilizar conhecimentos, atitudes e capacidades de pensamento críticos" (CRUZ; SANTOS, 2016).

Somam-se a estes estudos os trabalhos de Oldoni, Leite e Felipe (2018), Moebus e Martins (2013), Ferreira e Leite (2016), Schwan, Malesczyk e Wenzel (2017) que contemplaram no corpus de pesquisa, por ordem, 6, 48, 25 e 5 artigos, entre fontes de busca livre, períodos nacionais, ENEQ, ENPEC, respectivamente, abrangendo o período total de 2000 a 2015.

Finalmente, cabe salientar que, apesar dos esforços, durante a pesquisa não foi encontrado estudos anteriores comparando as características bibliográficas da temática proposta a partir do Google Acadêmico. 


\section{MÉtodo}

Trata-se de uma pesquisa descritiva bibliográfica, do tipo bibliométrica, de abordagem quantitativa, com amostragem não probabilística de julgamento e análise frequencial simples da ocorrência dos dados.

As pesquisas descritivas bibliográficas são aquelas que objetivam investigar determinadas características de uma população ou de certo fenômeno a partir de material já elaborado, principalmente, em livros e artigos (GIL, 2002). A amostragem não probabilística de julgamento ou intencional é aquela que busca os participantes que estariam na condição exigida (BAPTISTA; CAMPOS, 2016). A bibliometria é um dos métodos de coleta de dados quantitativos mais utilizados para investigar os padrões de publicações dentro de um determinado campo (SLUTSKY; AYTAC, 2016).

O universo de artigo compreendeu o total de trabalhos encontrados no Google Acadêmico onde a expressão "Alfabetização Científica" ocorresse no título (661 entradas), sendo 450 o total de trabalhos que compõe a amostra desta pesquisa, depois da exclusão de entradas apenas do tipo citação.

As etapas da pesquisa podem assim ser divididas:

a) acesso à base de dados do Google Acadêmico, realizando a busca de trabalhos a partir do descritor "Alfabetização Científica" e aplicação dos filtros de pesquisa: allintitle, não inclusão de citações e publicação até o ano de 2017;

b) Download dos trabalhos e arquivamento em uma pasta de computador adequadamente nomeada;

c) leitura seletiva dos artigos armazenados com a extração de dados dos trabalhos com base em categorias definidas a priori - ano, local da publicação, autores, título, palavras-chave e resumo - por meio do Mendeley ${ }^{1}$;

d) análise quantitativa das distribuições de frequências das categorias, com a utilização do MAXQDA ${ }^{\circledR 2}$ e Microsoft $^{\circledR}$ Excel $^{\circledR 3}$. A sistematização das sínteses analíticas das informações fora organizada, prioritariamente, na forma de tabelas.

\footnotetext{
${ }^{1}$ Mendeley é um programa, produzido pela Elsevier, de gerenciamento e compartilhamento de referências bibliográficas com colaboração online.

2 MAX Qualitative Data Analysis (MAXQDA) é um software de análise de dados qualitativos e mistos de pesquisa. Oferece diversas ferramentas para a organização e análise de dados, incluindo, texto, áudio, imagem, vídeo e arquivos bibliográficos.

${ }^{3}$ Microsoft Excel é um editor de planilhas para computadores. Seus recursos incluem uma interface intuitiva e capacitadas ferramentas de cálculo e de construção de gráficos.
} 


\section{Resultados}

\subsection{ANÁliSE dOS TítUlOS}

A busca refinada permitiu acesso a 450 trabalhos, publicados entre os anos de 1997 a 2017. A nuvem de palavras contidas nos títulos dos artigos está representada na Figura 1, que demonstra, em linhas gerais, a frequência dos principais termos associados a Alfabetização Científica, tais como, ensino (184), educação (65), fundamental (56), tecnológica (51), formação (44) e professores (42).

Figura 1 - Nuvem de palavras encontradas nos títulos dos trabalhos analisados

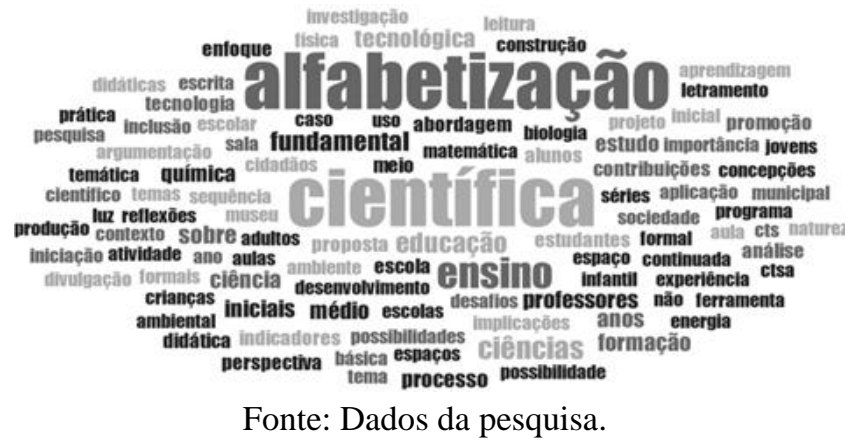

\subsection{Análise das Palavras-chave}

As palavras-chave representam os conceitos essenciais contidos em um texto e têm sido utilizadas como uma das principais estratégias de busca em banco de dados bibliográficos porque sintetizam e identificam o conteúdo do artigo (GONÇALVES, 2008).

Foram contabilizadas 1.374 palavras-chave em $84,2 \%$ dos 450 trabalhos, totalizando uma média de 3,62 palavras-chave por trabalho e desvio padrão 0,79. Foi identificado um total de 562 palavras distintas, sendo que 74,2\% delas aparecem uma única vez.

Tabela 1 - Distribuição das dez palavras-chave mais frequentes encontradas nos artigos (n=379)

\begin{tabular}{lcc}
\hline Palavras-chave & Quantidade & \% \\
\hline Alfabetização Científica & 263 & 69,4 \\
Ensino de Ciências & 86 & 22,7 \\
Alfabetização Científica e Tecnológica & 29 & 7,7 \\
Formação de Professores & 26 & 6,9 \\
Ensino de Química & 18 & 4,7 \\
CTS & 15 & 4,0 \\
Ensino Fundamental & 14 & 3,7 \\
Ensino Médio & 14 & 3,7 \\
Divulgação Científica & 13 & 3,4 \\
Ciência & 12 & 3,2 \\
Educação Ambiental & 12 & 3,2 \\
\hline
\end{tabular}

Fonte: Dados da pesquisa. 
A categorização a posteriori revelou que as palavras-chave expressam principalmente: a) alfabetização científica e/ou tecnológica; b) conteúdo / matéria escolar; c) adjetivação e/ou espaço educacional; e, d) nível e modalidade de educação e ensino.

Os resultados evidenciam que a média de palavras-chave nos trabalhos consultados observa a norma brasileira, mas há certa heterogeneidade e liberdade na seleção dos termos. Dessa maneira, pode ser sugerido o uso de vocabulário controlado na área de ensino de ciências e matemática como vem acontecendo em outras áreas.

\subsection{DiSTRIBUIÇÃO DE AUTORIA DAS PUBLICAÇÕES E EVOLUÇÃO HISTÓRICA}

Para conhecer que eram os autores com maior número de publicação, primeiramente, a autoria dos artigos foi desdobrada em registros individuais, resultando em 772 entradas autorais, dentre 1.052 registros, com média de 2,33 autores por trabalho e desvio padrão de 1,37. A Tabela 2 apresenta os resultados da distribuição da quantidade de autores por artigo.

Tabela 2 - Distribuição da quantidade de autores por artigo $(\mathrm{N}=450)$

\begin{tabular}{ccc}
\hline Número de autores por trabalho & Número de Artigos & \% \\
\hline $1-2$ & 297 & 66,0 \\
$3-4$ & 121 & 26,9 \\
$5-6$ & 24 & 5,3 \\
$7-8$ & 7 & 1,6 \\
$9-10$ & 1 & 0,2 \\
\hline Total & 450 & 100 \\
\hline
\end{tabular}

Fonte: Dados da pesquisa.

Com base nesta pesquisa, identificou-se que o artigo mais antigo com a expressão alfabetização científica no título foi o artigo de Gilberto Lacerda de 1997, intitulado "Alfabetização científica e formação profissional”, corroborando a literatura da área de ensino que aponta a década de 90 como o período que, no Brasil, se afloram as discussões sobre o conceito de alfabetização científica (LEAL; GOUVÊA, 2000).

A Figura 2 mostra a evolução quanto ao número de artigos da amostra pesquisada ao longo do tempo, observa-se que o número de publicação cresceu em tendência exponencial até o triênio 2012/2014 e que o período entre 2009/2011 e 2012/2014 apresentou a maior variação. 
Figura 2 - Evolução trienal das publicações sobre Alfabetização Científica, no período de 1997-2017, disponibilizadas no Google Acadêmico (em porcentagem)

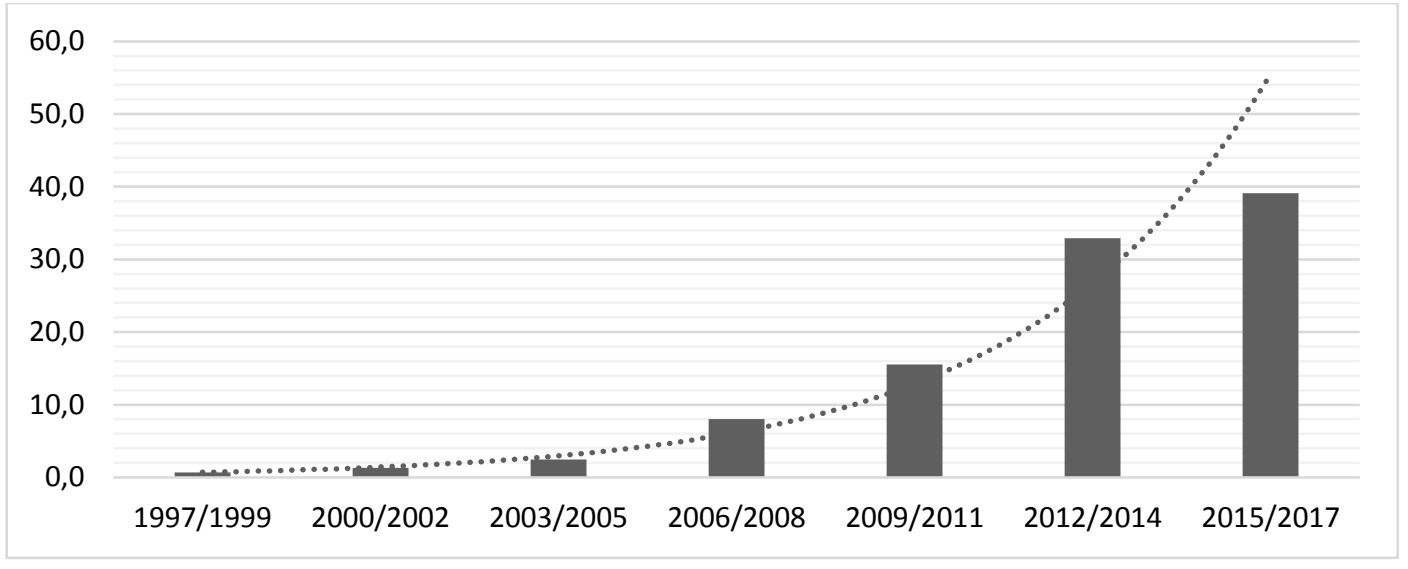

Fonte: Dados da pesquisa.

\subsection{PRinCipais AUtORES}

Verificou-se 772 autores distintos, dos quais 625 aparecem unicamente em um trabalho, sendo essa a faixa com maior representatividade $(59,41 \%)$, seguida de 98 autores em dois artigos $(9,31 \%)$. Na ordem inversa, 24 autores têm seus nomes em mais de quatro trabalhos $(0,38 \%)$ e apenas três em mais de 10 . Esse resultado revela uma alta variedade de autores que já abordaram a temática da alfabetização científica. Os 14 autores com maior número de artigos encontram-se na Tabela 3:

Tabela 3 - - Distribuição dos principais autores e respectivas afiliações por ordem de publicações $(\mathrm{N}=772)$

\begin{tabular}{lcc}
\hline \multicolumn{1}{c}{ Nome do Autor } & Afiliação & $\begin{array}{c}\text { Número de } \\
\text { artigos }\end{array}$ \\
\hline Lúcia Helena Sasseron & USP & 13 \\
Leonir Lorenzetti & UFPR & 12 \\
Augusto Fachín-Terán & UEA & 11 \\
Anna Maria Pessoa de Carvalho & USP & 9 \\
Marcelo Tadeu Motokane & USP & 9 \\
Martha Marandino & USP & 9 \\
Sidnei Quezada Meireles Leite & IFES & 9 \\
Maria das Graças Alves Cascais & SEMED/MANAUS & 7 \\
Fabiana Maris Versuti-Stoque & USP & 6 \\
Juliana Pinto Viecheneski & IFPA & 6 \\
Marcia Regina Carletto & UTFPR & 6 \\
Reginaldo dos Santos & UFPA & 6 \\
Rita de Cássia Frenedozo & UNICSUL & 6 \\
Rosemari Monteiro Castilho Foggiatto Silveira & UTFPR & 6 \\
\hline
\end{tabular}

Fonte: Dados da pesquisa.

Considerando os catorze autores que mais publicaram, a maior parte está vinculada à Universidade de São Paulo (USP). Semelhantemente, esse resultado foi achado também por Ferreira e Leite (2016) que ao realizarem uma pesquisa bibliográfica concernente aos trabalhos 
sobre Alfabetização Científica, publicados no Encontro Nacional de Ensino de Química (ENEQ), entre os anos de 2000 e 2014, identificaram a USP como a que mais apareceu nos trabalhos.

\subsection{LOCAIS DE PUBLICAÇÃO}

Quanto ao local de publicação, os resultados da pesquisa mostram significativa dispersão, numa média de 2,14 trabalho por fonte de publicação. No entanto, conforme apresentado na tabela 4, o Encontro Nacional de Pesquisa em Educação em Ciências (ENPEC), evento bienal realizado pela Associação Brasileira de Pesquisa em Ensino de Ciências (ABRAPEC), se destacou frente às outras 209 que foram listadas na pesquisa.

Tabela 4 - Distribuição dos principais locais de publicação. (N=210)

\begin{tabular}{lcc}
\hline \multicolumn{1}{c}{ Local } & Número & \% \\
\hline Encontro Nacional de Pesquisa em Educação em Ciências & 67 & 31,9 \\
Universidade de São Paulo & 13 & 6,2 \\
Simpósio de Educação em Ciências na Amazônia & 10 & 4,8 \\
Simpósio Nacional de Ensino de Ciência e Tecnologia & 10 & 4,8 \\
Congreso Internacional sobre Investigación en Didáctica de las Ciencias & 9 & 4,3 \\
Salão de Iniciação Científica da UFRGS & 8 & 3,8 \\
Universidade Tecnológica Federal do Paraná & 8 & 3,8 \\
Encontro Nacional de Didática e Práticas de Ensino & 7 & 3,3 \\
Universidade Federal de Santa Catarina & 7 & 3,3 \\
Encontro Nacional de Ensino de Química & 6 & 2,9 \\
\hline
\end{tabular}

Fonte: Dados da pesquisa.

Esse resultado está em acordo com a literatura, que tem apontado o ENPEC como um dos eventos científicos mais representativos para a socialização da pesquisa em educação em ciências. O evento, que ocorre desde 1997, está em sua décima segunda edição, congregando uma grande variedade de trabalhos produzidos na área (SLONGO; LORENZETTI; GARVÃO, 2015).

\section{CONCLUSÃo}

Por meio do presente estudo foi possível levantar, as principais palavras-chave, autores, evolução histórica e fontes de publicação sobre a temática Alfabetização Científica.

Após analisar os dados, identificou-se que houve um salto nas publicações de trabalhos sobre a temática a partir dos anos 2000. É expressiva a multiplicidade de fontes onde os trabalhos de alfabetização científica vêm sendo publicados, em contraste, a baixa tradição de publicação em relação aos autores, considerando a significativa quantidade de autores 
associados a apenas um único artigo. Ainda, o conjunto de palavras-chave extraídas apresentou alta variedade.

As limitações desse estudo dão-se pelo uso da amostragem intencional. Dessa maneira, os resultados expressam-se restritamente à base de dados selecionada, no caso, o Google Acadêmico.

Sugere-se que pesquisas futuras sejam realizadas no sentido de identificar quais são as principais referências adotadas pelos autores que produzem sobre a temática da Alfabetização Científica, bem como, quais abordagens didático-metodológicas são mais utilizadas nesses trabalhos. Pesquisas futuras também podem selecionar outras bases de dados para fins comparativos, analisar a rede de colaboração de autores ou verificar a aplicação das teorias bibliométricas.

Por fim, espera-se que os resultados deste estudo possam ter um impacto positivo sobre o futuro da pesquisa bibliométrica quanto à Alfabetização Científica.

\section{REFERÊNCIAS}

AULER, Décio; DELIZOICOV, Demétrio. Alfabetização científico-tecnológica para quê? Ensaio: Pesquisa em Educação em Ciências, Belo Horizonte, v. 3, n. 1, p. 122-134, 2001. Disponível em: http://www.scielo.br/pdf/epec/v3n2/1983-2117-epec-3-02-00122.pdf. Acesso em: 10 jun. 2019.

BAPTISTA, Makilim Nunes; CAMPOS, Dinael Corrêa de. Metodologias de pesquisa em ciências: análise quantitativa e qualitativa. 2. ed. Rio de Janeiro: LTC, 2016.

BRANDI, Arlete Terezinha Esteves; GURGEL, Célia Margutti do Amaral. A alfabetização científica e o processo de ler e escrever em séries iniciais: emergências de um estudo de investigação-ação. Ciência \& Educação, Bauru, v. 8, n. 1, p. 113-125, 2002. Disponível em: http://www.scielo.br/pdf/ciedu/v8n1/09.pdf. Acesso em: 12 jun. 2019.

BYBEE, Rodger W. Achieving scientific literacy. The Science Teacher, Arlington, v. 62, n. 7, p. 28-33, 1995.

CHASSOT, Attico. Alfabetização científica: uma possibilidade para a inclusão social.

Revista Brasileira de Educação, n. 22, p. 89-100, 2003. Disponível em: http://www.scielo.br/pdf/rbedu/n22/n22a09.pdf. Acesso em: 05 ago. 2018.

CRUZ, Joniely Cheyenne Moura; SANTOS, Paula Tauana. O estado do conhecimento em literacia a partir da base de dados Web Scopus na grande área das ciências sociais e humanidades - 2005 a 2015. In: Encontro Internacional de Formação de Professores e Fórum Permanente de Inovação Educacional, v. 9, n. 1, 2016. Anais [...]. Disponível em: https://eventos.set.edu.br/index.php/enfope/article/view/2502/832. Acesso em: 10 jun. 2019.

FERREIRA, Matheus; LEITE, Rosana Franzen. Alfabetização Científica e Ensino de Química: em análise, publicações do ENEQ entre os anos de 2000 e 2014. In: Encontro 
Nacional de Ensino de Química (ENEQ), XVIII, 2016. Anais [...]. Disponível em: http://www.eneq2016.ufsc.br/anais/resumos/R0915-1.pdf. Acesso em: 10 jun. 2019.

FOUREZ, Gérard. A construção das Ciências: introdução à filosofia e à ética das ciências. São Paulo: editora UNESP, 1995.

GARRIDO, Renato. Alfabetização científica. Universidade Federal do Rio Grande do Sul. [Monografia de Graduação]. Curso de Biblioteconomia. Universidade Federal do Rio Grande do Sul. 2010.

GIL, Antônio Carlos. Como elaborar projetos de pesquisa. 4 ed. São Paulo: Atlas, 2002.

GONÇALVES, Aline Lima. Uso de resumos e palavras-chave em Ciências Sociais: uma avaliação Encontros Bibli: revista eletrônica de biblioteconomia e ciência da informação, v. 13, n. 26, p. 78-93, 2008. Disponível em: https://doi.org/10.5007/1518-2924.2008v13n26p78. Acesso em: 10 jun. 2019.

HARZING, Anne-Wil; WAL, Ron van der. Google Scholar as a new source for citation analysis. Ethics in Science and Environmental Politics. v. 8 n. 1, p. 61-73, 2008. Disponível em: https://doi.org/10.3354/esep00076. Acesso em: 10 jun. 2019.

KLEIMAN, Angela B. Modelos de Letramento e as Práticas de Alfabetização na Escola, In: KLEIMAN, Angela B. (org.). Os Significados do Letramento - Uma nova perspectiva sobre a prática social da escrita, Campinas: Mercado das Letras, 1995.

KOUSHA, Kayvan; THELWALL, Mike; REZAIE, Somayeh. Assessing the citation impact of book-based disciplines: the role of Google Books, Google Scholar and Scopus. Journal of the American Society for Information Science and Technology, v. 62, n. 11, p. 2147-2164, 2011. Disponível em: https://doi.org/10.1002/asi.21608. Acesso em: 14 jun. 2019.

LEAL, Maria Cristina; GOUVÊA, Guaracira. Narrativa, mito, ciência e tecnologia: o ensino de ciências na escola e no museu. Ensaio: Pesquisa em Educação em Ciências (Belo Horizonte), v. 2, n. 1, p. 5-33, 2000. Disponível em: http://www.scielo.br/pdf/epec/v2n1/1983-2117-epec-2-01-00005.pdf. Acesso em: 14 jun. 2019.

LORENZETTI, Leonir; DELIZOICOV, Demétrio. Alfabetização científica no contexto das séries iniciais. Ensaio: Pesquisa em Educação em Ciências, Belo Horizonte, v. 3, n. 1, p. 3750, março, 2001. Disponível em: http://www.scielo.br/pdf/epec/v3n1/1983-2117-epec-3-0100045.pdf. Acesso em: 07 jul. 2019.

MEHO, Lokman I., YANG, Kiduk. Impact of data sources on citation counts and rankings of LIS faculty: Web of science versus Scopus and google scholar. Journal of the American Society for Information Science and Technology, v. 58, n. 13, p. 2105-2125, nov., 2007. Disponível em: https://doi.org/10.1002/asi.20677. Acesso em: 10 jun. 2019.

MION, Rejane Aurora; ANJOS, Enio dos; PIAZZETTA, Rubyan Lucas Santos. Estado da Arte sobre alfabetização e letramento científico-tecnológico na formação inicial de professores e ensino de. In: Encontro da rede de professores, pesquisadores e licenciandos de Física e de Matemática, 2010, São Carlos - SP, 2010. Anais [...]. Disponível em: http://www.enrede.ufscar.br/participantes_arquivos/E1_mion_IC.pdf. Acesso em: 10 jun. 2019. 
MOEBUS, Renata; MARTINS, Isabel. Leitura e alfabetização científica nas aulas de ciências: uma revisão de artigos publicados entre 2008 e 2012. In: Encontro Nacional de Pesquisa em Educação em Ciências, IX, 2013. Anais [...]. Disponível em:

http://www.nutes.ufrj.br/abrapec/ixenpec/atas/resumos/R0996-1.pdf. Acesso em: 10 jun. 2019.

OLDONI, Josiani Fátima Weimer Baierle; LEITE, Rosana Franzen; FELIPE, Lucas Mateus. Análise de artigos científicos nacionais sobre a alfabetização científica. In: Seminário Internacional de Pesquisa e Estudos Qualitativos, V, 2018. Anais [...]. Disponível em: https://sepq.org.br/eventos/vsipeq/documentos/07851985986/10. Acesso em: 10 jun. 2019.

PRAIA, João; GIL-PÉREZ, Daniel; VILCHES, Amparo. O papel da natureza da ciência na educação para a cidadania. Ciência \& Educação, v. 13, n. 2, p. 141-156, 2007. Disponível em: http://www.scielo.br/pdf/ciedu/v13n2/v13n2a01.pdf. Acesso em: 25 jun. 2019.

SASSERON, Lúcia Helena; CARVALHO, Anna Maria Pessoa de. Alfabetização científica: uma revisão bibliográfica. Investigações em Ensino de Ciências, Porto Alegre - RS, v. 16, n. 1, p. 59-77, 2011. Disponível em: https://www.if.ufrgs.br/cref/ojs/index.php/ienci/article/view/246/172. Acesso em: 14 jun. 2019.

SCHWAN, Fernanda; MALESCZYK, Clésio Rafael; WENZEL, Judite Scherer. A importância da alfabetização científica no ensino de ciências e química. In: Encontro de Debates sobre o Ensino de Química, 37º 2017. Anais [...]. Disponível em:

https://edeq.furg.br/images/arquivos/trabalhoscompletos/s05/ficha-168.pdf. Acesso em: 14 jun. 2019.

SLONGO, Iône Inês Pinsson; LORENZETTI, Leonir; GARVÃO, Marzane. A pesquisa em educação em ciências disseminada no ENPEC (2007 a 2013): explicitando dados e analisando tendências. In: Encontro Nacional de Pesquisa em Educação em Ciências, X, 2015, Águas de Lindóia: ABRAPEC, 2015. Anais [...]. Disponível em: http://www.abrapecnet.org.br/enpec/xenpec/anais2015/resumos/R1075-1.PDF. Acesso em: 03 jul. 2019.

SLUTSKY, Bruce; AYTAC, Selenay. Bibliometric Analysis and Comparison of Two STE. Science \& Technology Libraries, 35:2, 152-171, 2016. Disponível em: https://doi.org/10.1080/0194262X.2016.1171191. Acesso em: 25 jun. 2019.

SOARES, Magda. Letramento: um tema em três gêneros. 2. ed. Belo Horizonte: Autêntica, 2000 .

TEIXEIRA, Francimar Martins. Alfabetização científica: questões para reflexão. Ciência \& Educação, Bauru, v. 19, n. 4, p. 795-809, 2013. Disponível em: http://www.scielo.br/pdf/ciedu/v19n4/v19n4a02.pdf. Acesso em: 09 jul. 2019.

VIECHENESKI, Juliana Pinto; LORENZETTI, Leonir; CARLETTO, Marcia Regina. A alfabetização científica nos anos iniciais: uma análise dos trabalhos apresentados nos ENPECs. In: Encontro Nacional de Pesquisa em Educação em Ciências, X ENPEC, Águas de Lindóia, 2015. Anais [...]. Disponível em: http://www.abrapecnet.org.br/enpec/Xenpec/anais2015/resumos/R0409-1.PDF. Acesso em: 10 jul. 2019.

Recebido em: 10 de junho de 2019. Aprovado em: 19 de dezembro de 2019. 